inflammatory arthritis (IA), are one of the most common causes for presenteeism. There are no "gold standard" methods to identify, measure, or value the impact of presenteeism. Some evidence suggests the impact of presenteeism can be indirectly estimated using measures of health status and well-being.

Objectives: To explore whether selected measures of health status and wellbeing, commonly used in economic evaluations are conceptually useful to capture those aspects of IA that are associated with presenteeism.

Methods: A sample of individuals, aged 18 years and above, working in the UK with rheumatoid arthritis (RA), ankylosing spondylitis (AS), or psoriatic arthritis (PsA), was recruited via patient support groups. Semi-structured telephone interviews were designed to understand if, and how, RA, AS or PsA affects an individual's ability to work. Framework Analysis Methods were used and coding involved deductive and inductive approaches. A deductive approach was used to derive potential themes from measures of health status [EuroQol-5 Dimension- 5 level (EQ5D-5L) and Short Form 6 Dimension (SF6D)], and well-being [(ICEpop CAPability measure for Adults (ICECAP-A)]. An inductive approach was used to generate other themes not captured by these measures.

Results: Twenty-two employed individuals with RA $(n=10)$, AS $(n=9)$ or PsA $(n=3)$ were interviewed; $82 \%$ were female and, of the 22 patients, $23 \%$ had a manual job. The majority of interviewees explained that symptoms of the conditions increase levels of presenteeism, including: pain; stiffness; fatigue; emotional mental health; mental clarity. These symptoms make completing activities at work difficult, which, in turn, affects an individual's capability to maintain a successful career. The ICECAP-A was found to be a useful measure to capture the overall impact of presenteeism resulting from RA, AS or PsA. The SF6D and EQ5D were more specific measures capturing particular symptoms and activities that increase levels of presenteeism (see Table 1).

Table 1

\begin{tabular}{lccc}
\hline & \multicolumn{3}{c}{ Measure } \\
\cline { 2 - 4 } Theme & ICECAP-A & SF6D & EQ5D \\
\hline Achievement /Progress & $\mathrm{x}$ & & \\
Decisions & $\mathrm{x}$ & $\mathrm{x}$ & \\
Energy & $\mathrm{x}$ & & \\
Independence & $\mathrm{x}$ & $\mathrm{x}$ & $\mathrm{x}$ \\
Mental Health & $\mathrm{x}$ & $\mathrm{x}$ & $\mathrm{x}$ \\
Mobility & $\mathrm{x}$ & $\mathrm{x}$ & $\mathrm{x}$ \\
Pain & $\mathrm{x}$ & & \\
Self-care & $\mathrm{x}$ & & \\
Settled /Secure & $\mathrm{x}$ & $\mathrm{x}$ & $\mathrm{x}$ \\
Social Interaction & $\mathrm{x}$ & $\mathrm{x}$ & \\
Support & $\mathrm{x}$ & & \\
Usual Activities & $\mathrm{x}$ & & \\
Vigorous Activities & & &
\end{tabular}

Two further themes were identified using inductive methods: mental clarity and feeling understood. The effect of mental clarity or feeling understood is not captured by any of the domains in EQ-5D-5I or SF6D. The ICECAP-A is potentially able to capture the impact of these themes in the respective domains ability to achieve and progress and ability to gain support.

Conclusions: This study suggests that three existing measures (EQ5D, SF6D, and ICECAP-A) were successful, in different degrees, to capture the impact of presenteeism that result from the aspects and symptoms of IA. Potentially, these measures may be used in economic evaluations to capture the impact of presenteeism.

Acknowledgements: This work was supported by Arthritis Research UK and the Medical Research Council [20665]

Disclosure of Interest: None declared

DOI: 10.1136/annrheumdis-2017-eular.1803

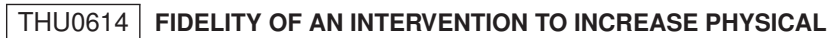 ACTIVITY IN OLDER ADULTS WITH CHRONIC PAIN: ANALYSIS OF AUDIO-RECORDED CONSULTATIONS FROM THE IPOPP PILOT TRIAL}

C. Jinks ${ }^{1}$, J. Proctor ${ }^{1}$, E.L. Healey ${ }^{1}$, J. McBeth ${ }^{2}$, C.A. Chew Graham ${ }^{1}$ on behalf of iPOPP team. ${ }^{1}$ Research Institute for Primary Care \& Health Sciences, Keele University, Keele; ${ }^{2}$ Arthritis Research UK Centre for Epidemiology, University of Manchester, Manchester, United Kingdom

Background: Physical activity levels are low in people $>65$ years and those with chronic musculoskeletal pain, yet increased physical activity can lead to reduced pain. Walking is acceptable to older people with pain, but interventions to increase walking in this population are untested. The PPOPP pilot trial investigated feasibility and acceptability of a walking intervention delivered by trained Health Care Assistants (HCAs) in 4 general practices in an individually randomised three arm trial (usual primary care, pedometer only and iPOPP, $n=50$ each arm). The iPOPP intervention comprised two consultations (1 week apart) and 8 weekly follow-up prompts (postcard, email or text).

Objectives: To investigate fidelity of the IPOPP intervention to inform design of a full-scale randomised controlled trial.

Methods: HCAs were asked to record 6 consultations ( 3 participants, first and second consultation). A fidelity checklist was developed (aligned to HCA training) and included activities expected (pedometer, user guide, walking diary, pain toolkit given; discussion of walking behaviour and barriers; action planning to develop walking goals; review of progress; positive feedback; revision of goals; relapse prevention strategies; and, preference for weekly prompts). Activities were scored as "Yes" (completed as intended), "partial" (some evidence), "No" (no evidence) or "NA" (not applicable). The checklist was applied to one recording (JP, CCG, CJ). Minor amendments clarified interpretation of checklist headings before application to 3 more recordings (JP, CCG). JP then scored remaining recordings $(n=14)$. Results: 3 HCAs recorded 18 consultations ( 9 first, 9 second). Most first consultations did not use the allocated 30 minutes (average 14 minutes). The pedometer, user guide, walking diary and pain toolkit were all given but there was a lack of explanation of the pain toolkit. Evidence of the benefits of walking on pain was well delivered $(\mathrm{N}=8)$, motivators to walk and goals were discussed in all 9, with goals set in 7 . Barriers to walking were not always discussed $(N=4)$ exploration of maintenance strategies lacking, especially with those patients who perceived themselves as already physically active. All HCAs arranged a second consultation within one week which were brief (average 6.5 minutes) and focused mainly on use of the pedometer. Patient goals were partially re-visited $(\mathrm{N}=7)$ and barriers to walking partially addressed $(\mathrm{N}=5)$. Maintenance strategies were not discussed ( $\mathrm{N}=8$ ).

Conclusions: Core iPOPP intervention components were well delivered. However we have identified areas to optimise ahead of a full-scale trial (how to discuss barriers to walking and maintenance strategies, development of motivational interviewing skills to support revision of goals, HCA knowledge of local activities). These findings will be triangulated with data from interviews with HCAs and participants to provide further evidence of the feasibility and acceptability of the iPOPP intervention.

Acknowledgements: Keele Clinical Trials Unit, Keele University supported the trial. ELH, CJ, and CCG are part funded by the NIHR Collaborations for Leadership in Applied Health Research and Care West Midlands. The views expressed in this paper are those of the author(s) and not necessarily those of the NHS, the NIHR, or the Department of Health.

Disclosure of Interest: None declared

DOI: 10.1136/annrheumdis-2017-eular.3232

\section{THU0615 PARTICIPANTS' EXPERIENCE OF THE MAKING IT WORK PROGRAM, AN ONLINE PROGRAM TO HELP PEOPLE WITH INFLAMMATORY ARTHRITIS REMAIN EMPLOYED}

A. $\mathrm{Li}^{1}$, C. Backman ${ }^{1,2}$, B. van As ${ }^{1}$, P. Rogers ${ }^{1}$, M. Gignac ${ }^{3,4}$, L. Li $^{1,2}$, J. Esdaile ${ }^{1,2}$ D. Lacaille ${ }^{1,2} .{ }^{1}$ Arthritis Research Canada, Richmond; ' University of British Columbia, Vancouver; ${ }^{3}$ University of Toronto; ${ }^{4}$ Institute of Work and Health, Toronto, Canada

Objectives: Health services addressing employment needs for people with arthritis are lacking. To address this need, we developed the Making it Work (MiW) program, an online self-management program aimed at helping people with inflammatory arthritis (IA) deal with employment issues. As part of a randomized controlled trial evaluating program effectiveness, this study reports on participants' experiences with various aspects of the program.

Methods: All participants in the MiW program between Jan20 and Dec7 2016 were included. Participants were recruited from rheumatologist practices, outpatient arthritis programs, a national consumer organization (Arthritis Consumer Experts), and community advertisements in BC, Alberta and Ontario. Eligibility criteria included: having IA; being currently employed; age 18-59 yrs; concern about their ability to work; and access to a computer. The program consisted of 5 online self-learning modules, 5 online group meetings facilitated by a vocational counsellor, an individual ergonomic assessment by an occupational therapist and an online session with a vocational counsellor. Feedback questionnaires were administered online after participants completed the program. Descriptive analyses were performed.

Results: The sample included 69 participants [80\% female; mean (SD) age: 45.3 (10.5) yrs; 83\% Caucasian; 91\% with post-secondary education; 52\% with RA, AS: $19 \%$, SLE: $17 \%$; PsA: $12 \%$ ]. Overall, participants expressed satisfaction with the program with $94.2 \%$ agreeing $(69.6 \%$ strongly and $24.6 \%$ somewhat) they would recommend this program to someone they know. When asked to rate program components on a scale of $0-10$ where $0=$ not useful at all and $10=$ very useful, participants rated all components favourably: median [25Q;75Q] for online modules: 8 [7;10], with highest ratings for the fatigue module (rated 10 by $42 \%$ ); online group meetings: 9 [7.5;10]; ergonomic and VRC assessments: $8[7 ; 10]$ each. Although participants had 2 weeks between meetings to complete the module, $55 \%$ did the module the week of, and $42 \%$ the day before, the group meeting. Median time to complete each module was $60 \mathrm{~min} .81 \%$ enjoyed being able to listen to the information (somewhat or strongly agreed), although $35 \%$ stated they would have preferred to read the information than listen to a narrator. $74 \%$ expect to use the online modules again in the future. Participants were also satisfied with the online group meetings: $93 \%$ were very or somewhat satisfied with the group facilitation; $87 \%$ satisfied with the group dynamic; $84 \%$ comfortable with the online format. When asked to rate their online group meeting experience on a scale of 1 to 10 , median [25Q;75Q] ratings for: ability to follow group discussion was 10 [9;10]; getting to know other participants: 7 [7;10]; feeling listened to and understood 9 [8;10]; feeling that group was supportive 9 [7;10]. $20 \%$ said it was difficult for them to attend group meetings.

Conclusions: In general, participants were highly satisfied with all aspects of the 
MiW program. Our study demonstrates that self-management programs, including group meetings, can be successfully delivered using an online format, which facilitates wider dissemination, with greater convenience to patients, and at lower costs.

Disclosure of Interest: None declared

DOI: 10.1136/annrheumdis-2017-eular.5246

\section{THU0616 PATIENTS' PERCEPTION ON DISEASE PROGRESSION AND ADHERENCE TO BIOLOGIC THERAPY}

E. Cefai, D. Balzan, C. Mercieca, A.A. Borg. Rheumatology, Mater Dei Hospital, Msida, Malta

Background: Adherence to medication depends on several factors such as medication beliefs, psychosocial factors, illness beliefs and concerns. The consequences of non-adherence are not insignificant, both from the clinical and health economic aspects.

Objectives: To assess medication adherence of patients taking biologics who are in remission or who have low disease activity and patients' perception of disease progression.

Methods: Forty-four consecutive patients attending a dedicated biologic clinic and treated with TNF inhibitors were interviewed following their visit. Patients were asked about adherence to treatment, adverse effects, concerns about biologics and their perception of disease progression. Demographic and disease activity data were recorded.

Results: Forty-four patients (23 females, 21 males) participated in the survey (21 suffered from rheumatoid arthritis, 17 from ankylosing spondylitis and 6 from psoriatic arthritis). The mean age was 55.1 (SD 12.62) years. The mean DAS28 was 2.26 (SD 1.03) and the mean BASDAI was 4.21 (SD 2.1). Minimal or no pain was reported by $75 \%$, mild or no fatigue by $78 \%$ and $77 \%$ had no restriction of activities of daily living. Medication adherence was reported as high by $68 \%$ and moderate by $32 \%$. There were no patients who had a low level of adherence. $5 \%$ of patients admitted to be unaware of the need to omit their biologic when ill and $40 \%$ when requiring surgery.

One out of 4 patients reported to have experienced adverse events, most commonly infections. $19 \%$ claimed to be moderately or very concerned about the adverse effects. On further questioning about the severity of potential adverse effects, $45 \%$ were unsure as to what the severity might be. When asked about duration of therapy, $29 \%$ replied more than 10 years; whilst $71 \%$ were unsure on when, if ever, the biologic is going to be stopped. Patients were also asked whether they believed that the biologic had successfully stopped further joint damage and $68 \%$ replied yes, $9 \%$ replied no, whilst $23 \%$ were unsure. Of those who replied yes, this perception was based on the absence or reduction of symptoms.

Conclusions: In this cohort of patients with low disease activity or remission, a third of patients still reported sub-optimal adherence to TNF inhibitor use. The benefits of adherence to biologic therapies need to be reinforced at every visit and factors leading to non-adherence addressed. Adverse effects remain a major concern that needs to be addressed, even in patients who have been on biologics for many years and have sustained remission or low disease activity. A quarter of patients were unsure whether biologics have stopped joint damage. Patients' perception of joint damage progression needs to be explored to find ways of making it more understandable to patients.

Disclosure of Interest: None declared

DOI: 10.1136/annrheumdis-2017-eular.3221

\section{THU0617 INFORMATION ON DISEASE AND TREATMENT OF PATIENTS WITH RHEUMATIC DISEASES TREATED WITH SUBCUTANEOUS BIOLOGICALS: SOURCES AND SATISFACTION. RHEU-LIFE SURVEY}

F. de Toro ${ }^{1}$, E. Batlle ${ }^{2}$, C. González ${ }^{3}$, M. Calvo ${ }^{4}$, M. Arteaga $^{4}$, L. Cea-Calvo ${ }^{4}$. ${ }^{1}$ INBIC, A Coruña; ${ }^{2}$ D.Salud Sant Joan, Alicante; ${ }^{3}$ H.G.U.Gregorio Marañón; ${ }^{4}$ Medical Affairs, Merck Sharp \& Dohme, Madrid, Spain

Objectives: To know the sources by which patients with rheumatic diseases treated with subcutaneous (SC) biological drugs obtain information on their treatment, their satisfaction with the information received from the hospital staff and their perception of the degree of information they have.

Methods: RHEU-LIFE was a survey handed by 50 rheumatologists from Spanish outpatients hospital offices to patients on SC biological drugs. They completed the survey anonymously at home and returned it by pre-paid post-mail. They selected from a list their sources of information (being able to mark all their sources), their satisfaction with the information received from the hospital staff and whether they had sought additional information in other sources.

Results: The survey was handed to 1000 patients and 592 returned it $(59.2 \%$, mean age 51.7 years, $57.6 \%$ women). The source of information most frequently mentioned was the rheumatologist (table) and, infrequently, the nurse or the hospital pharmacist. $46.1 \%$ had received information on the SC biological only orally, $6.0 \%$ written and $45.2 \%$ in both ways. The percentages quite or very satisfied with the information received were $42.6 \%$ and $44.5 \%$ respectively, $10.6 \%$ felt "neither satisfied nor dissatisfied" and $2.1 \%$ and $0.2 \%$ dissatisfied or very dissatisfied. The \% quite/very satisfied was higher when the information came from the rheumatologist $(89.6 \%$ vs $59.6 \%$ when the rheumatologist was not mentioned as source of information, $p<0.001$ ), and also when information was given both oral and written $(92.8 \%$ vs $86.1 \%$ if only oral information was given, $\mathrm{p}=0.0125)$. Some $45.2 \%$ had sought information on the SC biological in sources different to the hospital staff, the \% being higher among younger patients ( $<43$ years: $65.2 \%$ : 43-52 years: $51.4 \%, 53-61$ years: $39.1 \%,>61$ years: $20.6 \%, p<0.001)$ those with higher education level (without studies: $14.3 \%$, primary: $31.6 \%$, secondary: $55.6 \%$, vocational: $59.5 \%$, university or higher degrees: $64.9 \%, p<0.001$ ), and in those treated with a first biological SC drug $(49.9 \%$, vs. $37.7 \%$ and $37.2 \%$ with a second or third or further, $\mathrm{p}=0.014)$.

Sources from which patients received information on the different treatments and on the biological drug (patients could mark more than one option)

\begin{tabular}{lcc}
\hline & General information & Information on the SC biological \\
\hline Rheumatologist & $91.9 \%$ & $91.0 \%$ \\
Nurse & $10.2 \%$ & $17.9 \%$ \\
Hospital pharmacist & $3.2 \%$ & $11.5 \%$ \\
Other & $5.3 \%$ & $3.6 \%$ \\
Do not remember & $1.2 \%$ & $0.8 \%$ \\
Did not receive any information & $0.8 \%$ & $1.7 \%$ \\
\hline
\end{tabular}

Conclusions: Patients with rheumatic diseases mention the rheumatologist as their main source of information. The \% who mentioned nurses or pharmacists was low, although the latter are very frequently visited for dispensing the drug. Satisfaction with the information received was high, but clearly lower when the rheumatologist did not inform the patient. Nearly half reported having sought information in sources other than the hospital staff.

Acknowledgements: Endorsed by ConArtritis (National Coordinator of Patients with Arthritis). Funded by Merck Sharp \& Dohme of Spain

Disclosure of Interest: None declared

DOI: 10.1136/annrheumdis-2017-eular.3214

\section{THU0618 THE FACTORS AFFECTING WORKPLACE AND HOUSEHOLD LIFE IN ANKYLOSING SPONDYLITIS: A MULTI-DIMENSIONAL STUDY}

B. Taser ${ }^{1}$, F.F. Ayhan ${ }^{1,2}$, P. Borman ${ }^{1,3}$, A. Karagöz ${ }^{1} .{ }^{1}$ Physical medicine \& rehabilitation, Ankara Training and Research Hospital, Ankara; ${ }^{2}$ Physical therapy \& rehabilitation, Uşak University, School of Health Sciences, Uşak; ${ }^{3}$ Physical medicine \& rehabilitation, Hacettepe Medical School, Ankara, Turkey

Background: Work disability (WD) is the final stage of work problems and may be prevented by effective treatment and ergonomic interventions in earlier stage of work productivity loss and work instability. Contextual factors, disease related factors and local social security systems may also affect WD.

Objectives: We aimed to determine the predictive factors of work productivity and work stability in Turkish patients with ankylosing spondylitis

Methods: One-hundred patients with ankylosing spondylitis (31 females and 69 males) were included into this study. Demographics, working state, Bath scores of disease activity, functional and radiologic state, quality of life, cardiopulmonary functions (echocardiography, exercise stress test and pulmonary function test) and general work impairments (work productivity impairment and work instability) were recorded. WPAI and AS-WIS were selected as work outcomes. The most predictive factors were analyzed in work productivity and work instability. SPSS 14.0 statistics (descriptives, pearson correlation, and stepwise regressions were used for statistical analyses.

Results: Thirty-two patients (mean age: 42,6 \pm 11.7 ) were unemployed. Unemployed patients showed more female, less educated, low disease activity, and low fitness profile. The percentages of absenteeism (WPAl-1), presenteeism (WPAI-2), regular activity impairment (WPAI-3) and overall work impairment (WPAI-4) were determined as $8,44, \% 47$, and $\% 37$, respectively, in employee group. When affecting factors assessed with multiple stepwise linear regression analysed; the only determinant for absenteeism (WPAI-1) was the working day loss due to ilness at last year. Chest mobility, annual income level, AS quality of life (ASQLL), work change and co-morbid diseases were the determinants of presenteeism (WPAI-2), regular activity impairment (WPAI-3) and overall work impairment (WPAI-4). The score of mean work instability (AS-WIS) was $11.5 \pm 5.8$, and $42.6 \%$ of patients had low and $57,4 \%$ of patients had moderate-high work instability. Multiple stepwise linear regression analysis showed that most predictive factors for work instability were regular activity impairment (WPAI-3) and AS-QoL. The factors affecting non-work status were older age, female sex and low annual income level in stepwise logistic regression.

Conclusions: The common predictive factor of work productivity and work stability was quality of life. For evaluation of work productivity; socioeconomic factors such as annual income level and frequent work change were determinative as well as clinical datas (chest expansion and comorbid diseases). We suggested both pharmacologic and nonpharmacologic interventions to improve quality of life should be enabled in early period to improve work productivity.

Disclosure of Interest: None declared

DOI: 10.1136/annrheumdis-2017-eular.2550 